\title{
Randomized controlled trials and neurosurgery: the ideal fit or should alternative methodologies be considered?
}

\author{
Alireza Mansouri, MD, ${ }^{1,2}$ Benjamin Cooper, MD, ${ }^{4}$ Samuel M. Shin, MD, ${ }^{4}$ and \\ Douglas Kondziolka, MD, MSc ${ }^{3,4}$ \\ 1Division of Neurosurgery, University of Toronto, Ontario, Canada; ${ }^{2}$ Department of Clinical Epidemiology and Biostatistics, \\ McMaster University, Hamilton, Ontario, Canada; ${ }^{3}$ Departments of Neurosurgery and ${ }^{4}$ Radiation Oncology, NYU Langone \\ Medical Center, New York University, New York, New York
}

OBJECTIVE Randomized-controlled trials (RCTs) are advocated to provide high-level medical evidence. However, in neurosurgery, there are barriers to conducting RCTs. The authors of this study sought to analyze the quality of neurosurgical RCTs since 2000 to determine the adequacy of their design and reporting.

METHODS A search of the MEDLINE and EMBASE databases (2000-2014) was conducted. The medical subject heading (MeSH) terms used in the search included: "neurosurgery" OR "neurosurgical procedure," "brain neoplasms," "infarction" and "decompression," "carotid stenosis," "cerebral hemorrhage," and "spinal fusion." These studies were limited to RCTs, in humans, and in the English language. The Consolidated Standards for Reporting of Trials (CONSORT) and Jadad scales were used to assess the quality of RCT design and reporting. The standardized median times cited (median citations divided by years since publication) were used to assess impact. A pragmatic-explanatory continuum indicator summary-based scale was used to assess the design of the studies as primarily pragmatic or explanatory.

RESULTS Sixty-one articles were identified, and the following subspecialties were the most common: vascular (23, $37 \%$ ), followed by functional neurosurgery and neurooncology (both $13,21 \%$ ). The following nations were the primary leaders in RCTs: US (25 studies, 41\%), Germany (8 studies, 13\%), and the United Kingdom (7 studies, 11\%). Median sample size was 100 (interquartile range [IQR] 41.5-279). The majority of the studies (40,66\%) had pragmatic objectives. The median number of times cited overall was 69 (IQR 20.5-193). The combined median CONSORT score was 36 (IQR 27.5-39). Blinding was most deficiently reported. Other areas with a relatively low quality of reporting were sample size calculation ( $34.2 \%$ of surgical, $38.5 \%$ of drug, and $20 \%$ of device studies), allocation concealment $(28.9 \%$ of surgical, $23.1 \%$ of drug, and $50 \%$ of device studies), and protocol implementation (18.4\% of surgical, $23 \%$ of drug, and $20 \%$ of device studies). The quality of reporting did not correlate with the study impact. All studies had a median Jadad score $\leq 3$. Thirty-three pragmatic studies $(83 \%)$ and 5 explanatory studies $(25 \%)$ met the design objectives. All pragmatic studies based on drug and device trials met their objectives, while $74 \%$ of pragmatic surgical trials met their objectives.

CONCLUSIONS The prevalence of neurosurgical RCTs is low. The quality of RCT design and reporting in neurosurgery is also low. Many study designs are not compatible with stated objectives. Pragmatic studies were more likely to meet design objectives. Given the role of RCTs as one of the highest levels of evidence, it is critical to improve on their methodology and reporting.

http://thejns.org/doi/abs/10.3171/2014.12.JNS142465

KEY WORDS randomized controlled trial; trial design; publication; CONSORT

\footnotetext{
ABBREVIATIONS CONSORT = Consolidated Standards for Reporting of Trials; IQR = interquartile range; ISPOR = International Society for Pharmacoeconomics and Outcomes Research; JAMA = Journal of the American Medical Association; JNS = Journal of Neurosurgery; MeSH = medical subject headings; NASCET = North American Symptomatic Carotid Endarterectomy Trial; NEJM = New England Journal of Medicine; PCT = pragmatic RCT; PRECIS = pragmatic-explanatory continuum indicator summary; PROS = pragmatic registry-based observational studies; RCT = randomized controlled trial; STICH = Surgical Trial in Intracerebral Hemorrhage. ACCOMPANYING EDITORIAL See pp 552-557. DOI: 10.3171/2015.2.JNS142960.
}

SUBMITTED October 31, 2014. ACCEPTED December 30, 2014.

INCLUDE WHEN CITING Published online August 28, 2015; DOI: 10.3171/2014.12.JNS142465. 
$\mathrm{T}$ HE randomized-controlled trial (RCT) is considered to provide one of the highest levels of medical evidence. ${ }^{2}$ In particular, RCTs based on a large sample of participants are considered to be of even higher quality and have thus been favored by many epidemiologists. ${ }^{3,34}$ Since their establishment, RCTs have helped answer many important clinical questions through rigorous methods that reduce bias.

One of the pillars of an RCT is the presence of equipoise, which renders the conduct of treatment randomization ethical. In surgical specialties such as neurosurgery, however, decision-making is often based on the lessons taught by those in a position of authority or personal experience, ${ }^{32}$ considered to be Level III-V evidence. Therefore, among individuals, clear equipoise often does not exist. Other pertinent issues, such as the concept of the technical learning curve, standardization of a surgical intervention, and patient recruitment are also genuine concerns with surgical RCTs. ${ }^{17}$

Although a well-designed RCT has the potential to overcome many of these barriers, ${ }^{17}$ this goal cannot always be attained. In addition to significant resource requirements, rigorous trial design can potentially limit the applicability of its findings to the general population. Pragmatic RCTs (PCTs), as opposed to explanatory (mechanistic) trials, are designed to maintain the prognostic balance established by RCTs while permitting other aspects to proceed as routine. These types of trials represent an alternative strategy. ${ }^{36}$ Their flexible nature, however, has been shown to often result in an inadvertent breakdown of randomization, and thus decrease the value of the trial. ${ }^{6}$ Others suggest that these limitations should be accepted as realities. Any shortcomings can be remedied by implementing reporting criteria trials that can help readers identify the quality of the evidence. ${ }^{29}$ The Consolidated Standards of Reporting Trials (CONSORT) is one such example. ${ }^{19}$ Recent assessments of neurosurgical trials with regard to the quality of reporting using various scales, however, have noted that the overall quality is rather low but perhaps improving over time. ${ }^{13,27,33}$ This is likely an issue related to both a lack of awareness of these reporting criteria and the inherently flawed design of many neurosurgical RCTs.

While RCTs are and will continue to provide some of the highest level of evidence, certain scenarios call for alternative study design strategies. Rather than establish rigid guidelines of design and reporting to create a better match between neurosurgery and RCTs, it may be worthwhile to accept this difference and conduct clinical studies based on designs that reflect the constraints of a particular clinical question. Indeed, the "pragmatic" component of the phrase "pragmatic clinical trials" has served to emphasize the practical and sensible solution that PCT design offers to address some of the logistic barriers posed by the traditional RCT design. Furthermore, many fields in neurosurgery (such as benign primary tumors) have lacked the benefit of evidence-based medicine derived from RCTs, and current practice is, at the very best, based on prospective observational series. These studies are often limited by very small sample sizes, preventing definitive conclusions. Therefore, in certain clinical scenarios, the pragmatic approach to such shortcomings could perhaps be through the use of multicenter, registry-based collection of prospective observational data. Thus, pragmatic registry-based observational studies (PROS), designed through rigorous methodology, can serve as a viable substitute in clinical scenarios in which a well-designed and conducted RCT is simply not feasible. The collection of high-quality prospective data has been facilitated through the implementation of registries, contributing greatly to clinical data in a variety of medical specialties. ${ }^{7,22}$

In this study, we sought to identify the main surgical RCTs conducted with relevance to the field of neurosurgery since the year 2000 . Our objectives were to critically appraise the design and reporting of RCTs in neurosurgery, and to correlate the design of the study (whether explanatory or pragmatic) with its specific objectives to identify the extent of inconsistency. Furthermore, we outline potential strategies to improve the quality of the design and conduct of PROS for situations in which RCTs are not feasible.

\section{Methods \\ Search Strategy}

A search of the MEDLINE and EMBASE (2000 to the present) online databases was initiated on September 9, 2014, to identify RCTs in neurosurgery. The choice of the earlier date boundary was based on the demonstrated improvement in the indexing of studies in databases such as MEDLINE, as a result of the findings and recommendations by Dickersin et al. and Evans s $^{8,9}$ and to include a sampling of relatively recent studies. The search was restricted to the English-speaking literature and studies pertaining to humans only. The initial search contained the medical subject heading (MeSH) terms "neurosurgery" or "neurosurgical procedure" and the results were limited to "randomized controlled trials" as the publication type. An analysis of the results from this search suggested that some of the key neurosurgical RCTs had not been identified. Therefore, on September 19, 2014, the following MeSH terms were searched to identify additional studies: "brain neoplasms" (relevant to the surgical aspect of neurooncology), "infarction" and "decompression" (relevant to the overlap between the field of neurology, critical care, and neurosurgery), "carotid stenosis" (relevant to neurosurgeons involved in open and/or endovascular management of carotid disease), "cerebral hemorrhage" (relevant to the overlap between the field of neurology, critical care, and neurosurgery), and "spinal fusion" (primarily relevant to the influx of new devices into this subspecialty). Similar to before, the results for each of these categories were limited to "randomized controlled trials" as the publication type.

The search results were imported into EndNote (version X7.2) and duplicates were removed. Titles and abstracts were reviewed by 1 author (A.M.) to identify relevant neurosurgical studies. Incomplete studies, interim reports of longer trials, and published protocols without any pertinent patient data were excluded. In the case of studies published with varying follow-up durations or different analyses of the same trial, only the initial study was considered; this resulted in the exclusion of 9 studies from 
the final list. To focus the results of our analysis on the quality of reporting of RCTs pertaining to surgical interventions in neurosurgery, studies explicitly pertaining to physiotherapy, pain management, or anesthesia were not included. In "brain neoplasms," only studies assessing at least 1 form of neurosurgical intervention were included. Therefore, studies solely focused on chemotherapy or radiation-based therapies other than radiosurgery were not included. In "carotid stenosis" only studies assessing at least 1 type of carotid stenting or endarterectomy were included. In "cerebral hemorrhage," studies assessing solely the role of conservative measures such as cooling were not included. Lastly, in "spinal fusion," studies pertaining solely to pain management, rehabilitation, and physiotherapy were not included. These strategies were implemented to ensure that RCTs assessing a surgical intervention in at least 1 of the arms of the study were captured. A summary of the search strategy and results is summarized in Fig. 1.

Studies were categorized as "surgical," "drug," or "device," depending on the trial question. The following information was extracted from each individual trial: year of publication, journal name, country of the study's lead investigator, the subspecialty to which the trial pertained, the sample size, whether the study was funded by industry (if not clear, "unknown" was indicated), whether the study was a multicenter study, and the number of times the study was cited. The Web of Science electronic database was used to obtain the latter.

\section{Study Assessment}

The full text of the relevant studies was reviewed to determine the revised CONSORT ${ }^{19}$ and Jadad scores ${ }^{12}$ as an assessment of the quality of the reporting of these RCTs. For each of the 22 categories of the CONSORT scale, 2 points were given if the category was addressed fully, 1 point if only partially addressed, and 0 points if the category was not addressed at all; through this strategy, the range of possible CONSORT scores for a given study would be 0 to 44 . The CONSORT statement was reviewed by the 3 independent assessors (A.M., B.C., and S.M.S.) prior to scoring of studies to ensure a unified understanding of the headings and appropriate scoring strategy. For each category, the median score obtained by the 3 reviewers was used as the overall score. The Spearman's correlation analysis was used to assess the interobserver variability for the overall CONSORT scores. A correlation coefficient greater than 0.8 was considered to be satisfactory. One reviewer (A.M.) assessed the studies for the Jadad score. In this 3-category scale, 1 point was given if randomization was noted along with an additional point if the method chosen was appropriate; a point was deducted if the method chosen was inappropriate. Similarly, 1 point was given if blinding was noted, along with an additional point if the method chosen was appropriate; a point was deducted if the method chosen was inappropriate. For the final category, a point was given if all patients initially enrolled had been accounted for. Thus, the range of possible Jadad scores would be 0 to 5 .

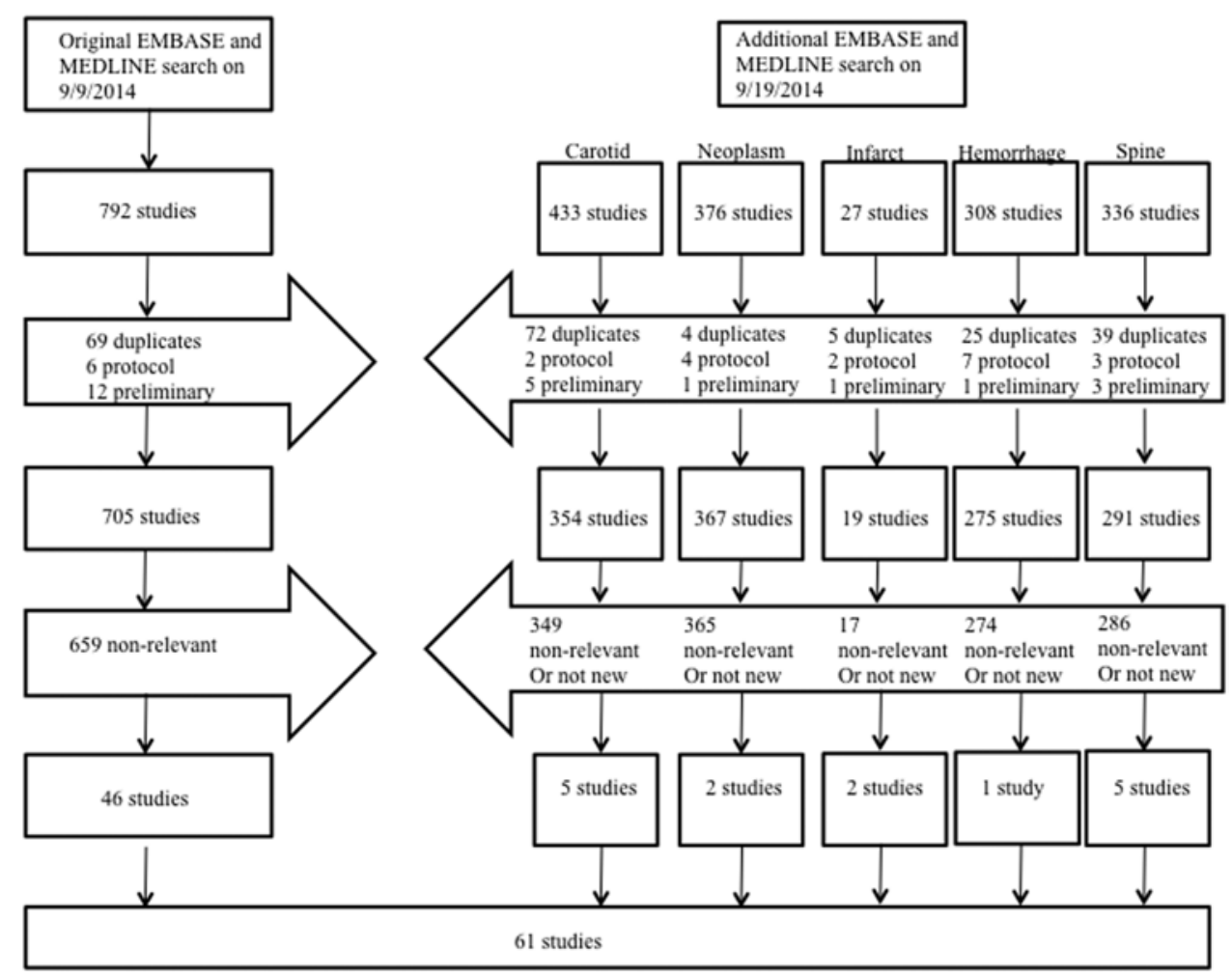

FIG. 1. Flow diagram outlining the algorithm of the MEDLINE and EMBASE search protocol. The search was conducted in a stepwise manner in the direction of the small arrows. Titles and abstracts were reviewed to exclude nonrelevant studies (boxes with arrows). 
The 2013 impact factors for the journals publishing the manuscripts identified by our search were identified through an open access website that compiles journal citation report factors for convenient access (http://www. impact-factor.org). Five of these results were selected at random and the impact factor values were confirmed with an independent search to assure the validity of the compiled data, which was found to be true. In 1 of the search results, the original journal Surgical Neurology changed names to World Neurosurgery; for this paper we used the 2009 impact factor, which was the latest available value. The Spearman's correlation analysis was used to determine the correlation of journal impact factor and the standardized median times cited on the total CONSORT score. The latter factor has been used previously as a surrogate measure for the overall impact of the particular RCT on the medical literature pertaining to the field. ${ }^{30}$ The Mann-Whitney U-test for nonparametric variables was used to determine a statistical difference between the median score for articles published in the top 3 neurosurgical journals compared with the top 3 medical journals. A $\mathrm{p}$ value $<0.05$ was considered statistically significant. The statistical program SPSS (version 22.0, IBM) was used for all statistical analyses.

The nature of the question for each trial was used by 1 author (A.M.) to determine whether the objective of the trial was explanatory or pragmatic. Subsequently, an arbitrary scale based on the 10-item pragmatic-explanatory continuum indicator summary (PRECIS) tool ${ }^{31}$ was used to determine the position of the study on the pragmatic-explanatory continuum as per its actual design. In this scale, all studies would have a starting score of 10 . A point was deducted if the design of the study was pragmatic for the given PRECIS item while a point was added if the design was explanatory for the given item. Thus, a fully pragmatic trial would have a score of 0 while a fully explanatory trial would have a score of 20 . While the original article by Thorpe and colleagues ${ }^{31}$ provides a comprehensive overview of the criteria used in this scoring strategy, a pragmatic trial would, in brief, be one wherein: 1) all potential participants are enrolled regardless of baseline risk (for example, no run-in phase has been implemented); 2) the experimental and comparator intervention can be applied according to the best judgment of the clinician without the need for specific expertise or training; 3 ) the primary outcome of interest can be objectively measured; 4) there are limited to no follow-up/compliance/protocol adherence assessments; and 5) the final analysis is made on an intent-to-treat basis. An example of a primarily pragmatic RCT is the International Surgical Trial in Intracerebral Hemorrhage (STICH) study ${ }^{18}$ in which all patients with a spontaneous supratentorial hemorrhage within the past 72 hours were eligible and the comparators were best medical therapy versus surgical evacuation of hematoma, both implemented according to the best judgment of the managing clinician. The outcome measured here was objective (death or disability based on the Glasgow Outcome Scale) and it was ascertained primarily through mailed questionnaires with or without confirmation of living status without the need for a formal follow-up. No formal assessment of compliance/adherence was conducted and the final
TABLE 1. Characteristics of RCTs identified in the search strategy

\begin{tabular}{lc}
\hline \multicolumn{1}{c}{ Variable } & Value \\
\hline Subspecialty (\%) & \\
Vascular & $23(38)$ \\
Functional & $13(21)$ \\
Oncology & $13(21)$ \\
Spine & $7(12)$ \\
Trauma & $2(3)$ \\
Pediatrics & $2(3)$ \\
Other & $1(2)$ \\
Study type (\%) & \\
Surgical & $38(62)$ \\
Drug & $13(21)$ \\
Device & $10(16)$ \\
Journal (\%) & \\
Neurosurgical & $20(33)$ \\
Nonneurosurgical & $41(67)$ \\
Source nations (\%) & \\
US & $25(41)$ \\
Germany & $8(13)$ \\
United Kingdom & $7(11)$ \\
France & $5(8)$ \\
The Netherlands & $5(8)$ \\
Canada & $3(5)$ \\
India & $2(3)$ \\
Others & $6(10)$ \\
Median sample size (\%) & \\
<100 patients & $32(52)$ \\
>100 patients & $29(48)$ \\
\hline Median times cited (IQR) & $69(20.5-193)$ \\
Median standardized times cited (IQR) & $10.3(4.0-32)$ \\
\hline Multicenter (\%) & \\
Yes & $45(74)$ \\
No & $16(26)$ \\
Industry funding (\%) & \\
Yes & $20(33)$ \\
No & $36(59)$ \\
Unknown & $5(8)$ \\
Study objective (\%) & \\
Pragmatic & $40(66)$ \\
Explanatory & $21(34)$ \\
\hline &
\end{tabular}

analysis was performed on an intent-to-treat basis. Note that the addition and subtraction of points in this scale is completely arbitrary and done solely to create a sense of the overall directionality for the given trial. The proportion of trial designs meeting the study objective were categorized by the study category.

\section{Results}

Upon conducting the outlined search of the literature, 61 suitable articles were identified (Table 1). The range of RCTs per year was roughly similar, averaging approximately 5 studies and peaking at 8 studies in 2007 (Fig. 2). Studies pertaining to vascular neurosurgery comprised 23 of the studies (37\%), followed by studies in functional neu- 


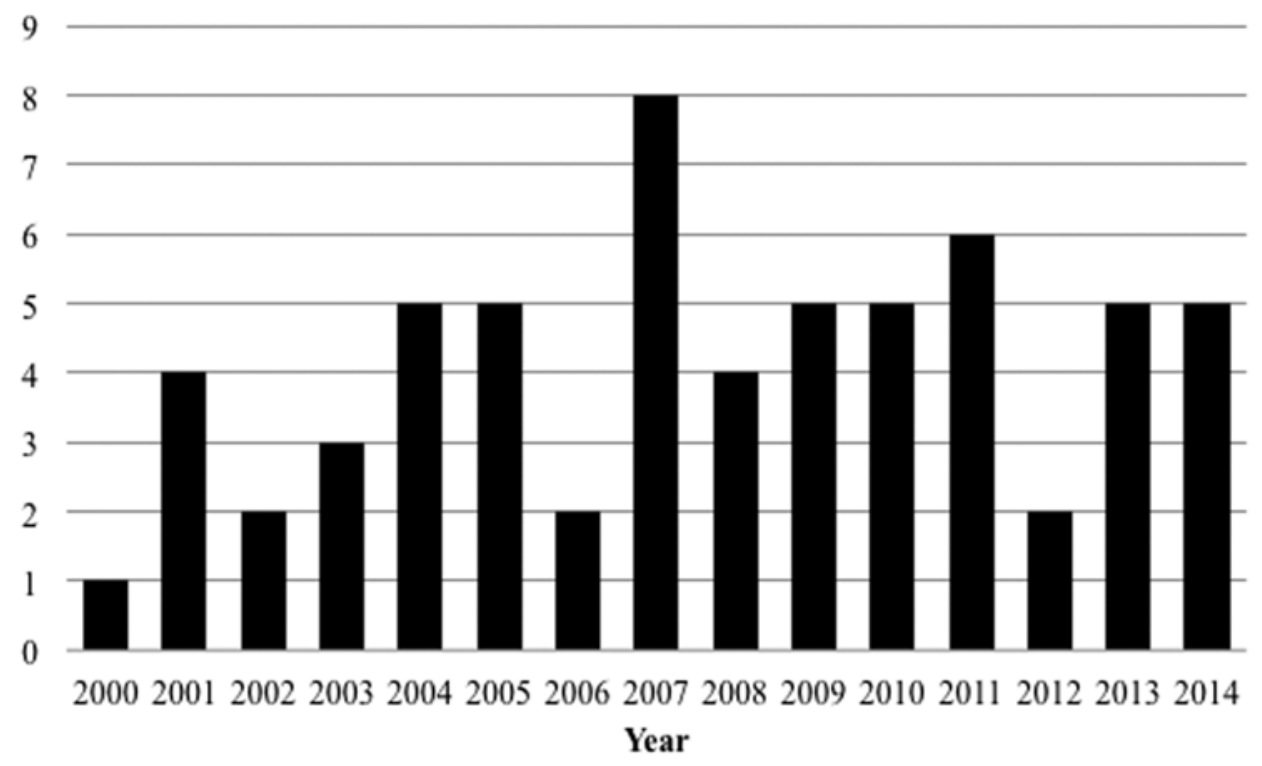

FIG. 2. Bar graph of the annual number of RCTs in neurosurgery based on the current search strategy, beginning in the year 2000 .

rosurgery and neurooncology, with 13 studies (21\%) each. The majority of RCTs in neurooncology were based on brain metastases and high-grade gliomas; low-grade primary tumors were drastically underrepresented. Surgical studies were found to be the most common type (38 studies, 62\%). One-third of the studies were published in neurosurgical journals such as the Journal of Neurosurgery $(J N S)$ with 5 studies (25\%), Neurosurgery with 4 studies (20\%), and Spine with 2 studies (10\%). Among the studies published in nonneurosurgical journals, $6(15 \%)$ were in The Lancet, 5 (12\%) were in The New England Journal of Medicine (NEJM), and $2(5 \%)$ were in The Journal of the American Medical Association (JAMA). The US (25 studies, $41 \%$ ), Germany (8 studies, $13 \%$ ), and the United Kingdom ( 7 studies, $11 \%$ ) were the top 3 nations contributing to this literature. The median sample size for all studies was 100 patients (interquartile range [IQR] 41.5-279); more than half of the studies contained a sample size smaller than 100 patients. The majority of the studies $(45,74 \%)$ were multicenter and not funded by industry $(36,59 \%)$. Based on the study questions, the majority of the studies $(40,66 \%)$ appeared to have pragmatic objectives. The median number of times cited overall was 69 (IQR 20.5-193).

Each study was assessed independently to identify the CONSORT score for each category. The interobserver agreement was strong (Spearman's correlation coefficients: A.M. and S.M.S. $=0.81$; A.M. and B.C. $=0.80$; B.C. and S.S. $=0.92)$. The combined median CONSORT score was 36 (IQR 27.5-39). The extent of fulfillment of each item in the CONSORT scale, based on study type, is summarized in Table 2. The areas with the greatest deficiency regarding quality of reporting pertained to blinding, in which a large proportion of studies did not fulfill any of the criteria; surgical studies, in particular, failed to report blinding in $65.8 \%$ of studies. Other areas with a relatively low quality of reporting with none of the criteria met pertained to the categories of sample size calculation and justification (34.2\% of surgical, 38.5\% of drug, and
$20 \%$ of device studies), allocation concealment (28.9\% of surgical, $23.1 \%$ of drug, and $50 \%$ of device studies), and protocol implementation (18.4\% of surgical, $23 \%$ of drug, and $20 \%$ of device studies). The majority of studies were of good quality with regard to reporting clearly defined eligibility criteria, study objectives, and statistical methods. In addition, the baseline characteristics of the patients were in general well-documented, along with a high frequency of reporting all adverse events.

Spearman's correlation coefficient between total CONSORT score and the journal impact factor was 0.66, reflecting a strong correlation. The top 3 medical journals, based on impact factor, were NEJM (54.42), Lancet (39.2), and JAMA (29.98; Table 3). The top 3 neurosurgical journals were JNS (3.2), Neurosurgery (3.0), and Spine (2.45). The median CONSORT score of articles published in these medical journals was significantly greater than that of studies published in the neurosurgical journals (Table 3 ). The standardized median times cited was not correlated with the median CONSORT score.

The major design components of all study types, based on the median score of the Jadad items of appropriateness of randomization and blinding methods, along with accounting for all participants, were weak across various types of trials, with each having a median Jadad score of 3 or less (Table 4). Among all study types, many of the trials either did not involve blinding or failed to implement the methods appropriately. Although the sample size of device studies may have been too low to draw firm conclusions, these study types appeared to be associated with the weakest design quality overall.

The median PRECIS-based score for studies with pragmatic objectives was 6 (IQR 4-6), and was 8 (IQR 6-11.5) for studies with explanatory objectives. While this difference was significant, a substantial amount of overlap between the scores was observed (Fig. 3 left). Among the 40 pragmatic studies, 33 (83\%) met the design objectives; 3 of these (9\%) were overall neutral (PRECIS-based 
TABLE 2. Percentage of neurosurgical RCTs meeting items in the CONSORT checklist*

\begin{tabular}{|c|c|c|c|}
\hline $\begin{array}{l}\text { CONSORT } \\
\text { Variable }\end{array}$ & $\begin{array}{l}\text { Surgical } \\
\text { Studies }\end{array}$ & $\begin{array}{c}\text { Drug } \\
\text { Studies }\end{array}$ & $\begin{array}{l}\text { Device } \\
\text { Studies }\end{array}$ \\
\hline $\begin{array}{l}\text { Median total score } \\
\quad(\mathrm{IQR})\end{array}$ & $32(23.8-35.2)$ & $32(29.5-34.5)$ & $26(24.5-33)$ \\
\hline \multicolumn{4}{|l|}{ Title \& abstract } \\
\hline All criteria & 71.0 & 61.5 & 70 \\
\hline Some criteria & 28.9 & 38.5 & 30 \\
\hline No criteria & 0 & 0 & 0 \\
\hline \multicolumn{4}{|c|}{ Introduction \& background } \\
\hline All criteria & 60.5 & 69.2 & 70 \\
\hline Some criteria & 39.5 & 30.8 & 30 \\
\hline No criteria & 0 & 0 & 0 \\
\hline \multicolumn{4}{|l|}{ Participants } \\
\hline All criteria & 86.8 & 76.9 & 80 \\
\hline Some criteria & 10.5 & 23.1 & 20 \\
\hline No criteria & 2.7 & 0 & 0 \\
\hline \multicolumn{4}{|l|}{ Interventions } \\
\hline All criteria & 55.3 & 69.2 & 50 \\
\hline Some criteria & 44.7 & 30.8 & 50 \\
\hline No criteria & 0 & 0 & 0 \\
\hline \multicolumn{4}{|l|}{ Objectives } \\
\hline All criteria & 89.5 & 92.3 & 90 \\
\hline Some criteria & 10.52 & 7.7 & 0 \\
\hline No criteria & 0 & 0 & 10 \\
\hline \multicolumn{4}{|l|}{ Outcomes } \\
\hline All criteria & 81.6 & 69.2 & 60 \\
\hline Some criteria & 15.8 & 30.8 & 30 \\
\hline No criteria & 2.6 & 0 & 10 \\
\hline \multicolumn{4}{|c|}{ Sample size calculations \& justifications } \\
\hline All criteria & 65.8 & 61.5 & 50 \\
\hline Some criteria & 0 & 0 & 30 \\
\hline No criteria & 34.2 & 38.5 & 20 \\
\hline \multicolumn{4}{|c|}{ Random sequence generation } \\
\hline All criteria & 52.6 & 69.2 & 50 \\
\hline Some criteria & 42.1 & 15.4 & 30 \\
\hline No criteria & 5.3 & 15.4 & 20 \\
\hline \multicolumn{4}{|c|}{ Allocation concealment } \\
\hline All criteria & 18.5 & 15.4 & 30 \\
\hline Some criteria & 52.6 & 61.5 & 20 \\
\hline No criteria & 28.9 & 23.1 & 50 \\
\hline \multicolumn{4}{|c|}{ Implementation of methods } \\
\hline All criteria & 63.2 & 38.5 & 0 \\
\hline Some criteria & 18.4 & 38.5 & 80 \\
\hline No criteria & 18.4 & 23.0 & 20 \\
\hline \multicolumn{4}{|l|}{ Blinding } \\
\hline All criteria & 7.9 & 0 & 30 \\
\hline Some criteria & 26.3 & 61.5 & 40 \\
\hline No criteria & 65.8 & 38.5 & 30 \\
\hline \multicolumn{4}{|l|}{ Statistical methods } \\
\hline All criteria & 81.6 & 69.2 & 50 \\
\hline Some criteria & 13.2 & 15.4 & 20 \\
\hline No criteria & 5.2 & 15.4 & 30 \\
\hline
\end{tabular}

TABLE 2. Percentage of neurosurgical RCTs meeting items in the CONSORT checklist* ${ }^{*}$ continued)

\begin{tabular}{|c|c|c|c|}
\hline $\begin{array}{l}\text { CONSORT } \\
\text { Variable }\end{array}$ & $\begin{array}{l}\text { Surgical } \\
\text { Studies }\end{array}$ & $\begin{array}{l}\text { Drug } \\
\text { Studies }\end{array}$ & $\begin{array}{l}\text { Device } \\
\text { Studies }\end{array}$ \\
\hline \multicolumn{4}{|l|}{ Participant flow } \\
\hline All criteria & 68.4 & 69.2 & 60 \\
\hline Some criteria & 23.7 & 15.4 & 40 \\
\hline No criteria & 7.9 & 15.4 & 0 \\
\hline \multicolumn{4}{|l|}{ Recruitment } \\
\hline All criteria & 23.7 & 30.8 & 50 \\
\hline Some criteria & 60.5 & 30.8 & 20 \\
\hline No criteria & 15.8 & 38.4 & 30 \\
\hline \multicolumn{4}{|l|}{ Baseline data } \\
\hline All criteria & 97.4 & 84.6 & 80 \\
\hline Some criteria & 2.6 & 15.4 & 10 \\
\hline No criteria & 0 & 0 & 10 \\
\hline \multicolumn{4}{|c|}{ Numbers analyzed } \\
\hline All criteria & 57.9 & 69.2 & 30 \\
\hline Some criteria & 42.1 & 23.1 & 70 \\
\hline No criteria & 0 & 7.7 & 0 \\
\hline \multicolumn{4}{|c|}{ Outcomes \& estimation of effect size \& precision } \\
\hline All criteria & 52.6 & 69.2 & 30 \\
\hline Some criteria & 47.4 & 23.1 & 60 \\
\hline No criteria & 0 & 7.7 & 10 \\
\hline \multicolumn{4}{|l|}{ Ancillary analyses } \\
\hline All criteria & 86.8 & 76.9 & 30 \\
\hline Some criteria & 10.5 & 7.7 & 30 \\
\hline No criteria & 2.7 & 15.4 & 40 \\
\hline \multicolumn{4}{|l|}{ Adverse events } \\
\hline All criteria & 81.6 & 61.5 & 90 \\
\hline Some criteria & 10.5 & 30.8 & 0 \\
\hline No criteria & 7.8 & 7.7 & 10 \\
\hline \multicolumn{4}{|l|}{ Data interpretation } \\
\hline All criteria & 55.3 & 84.6 & 70 \\
\hline Some criteria & 42.1 & 15.4 & 30 \\
\hline No criteria & 2.6 & 0 & 0 \\
\hline \multicolumn{4}{|l|}{ Generalizability } \\
\hline All criteria & 44.7 & 76.9 & 50 \\
\hline Some criteria & 47.4 & 15.4 & 50 \\
\hline No criteria & 7.9 & 7.7 & 0 \\
\hline \multicolumn{4}{|c|}{ Overall interpretation in the context of available evidence } \\
\hline All criteria & 78.9 & 84.6 & 70 \\
\hline Some criteria & 21.1 & 15.4 & 30 \\
\hline No criteria & 0 & 0 & 0 \\
\hline
\end{tabular}

* All data given as percentages unless otherwise indicated.

score of 10$)$, and $5(15 \%)$ had an overall design that was more consistent with an explanatory study. Among the 21 explanatory studies, 5 (24\%) met the design objectives; 4 $(19 \%)$ of these were overall neutral, and $11(52 \%)$ had an overall design that was more consistent with a pragmatic study (Fig. 3 right). Irrespective of the study type (surgical, drug, or device), a greater proportion of pragmatic studies were designed to meet their objectives as compared with the explanatory studies ( $80 \%$ vs 25\%; Fig. 3 right). All pragmatic studies based on drug and device trials met 
TABLE 3. Comparison of the medical and neurosurgical journals with the top 3 highest impact factors using the combined median CONSORT score

\begin{tabular}{lcccc}
\hline Journal & $\begin{array}{c}\text { Impact } \\
\text { Factor }\end{array}$ & $\begin{array}{c}\text { No. of } \\
\text { Studies } \\
\text { Contributed }\end{array}$ & $\begin{array}{c}\text { Journal } \\
\text { Median } \\
\text { CONSORT } \\
\text { Scores }\end{array}$ & $\begin{array}{c}\text { Combined } \\
\text { Median } \\
\text { CONSORT } \\
\text { Scores* }\end{array}$ \\
\hline NEJM & 54.42 & 5 & 38 & \\
Lancet & 39.2 & 6 & 38.5 & 39 \\
JAMA & 29.98 & 2 & 42 & \\
\hline JNS & 3.2 & 5 & 30.5 & \\
Neurosurgery & 3.0 & 4 & 30 & 30 \\
Spine & 2.45 & 2 & 33.5 & \\
\hline
\end{tabular}

* The combined median CONSORT score of the top 3 medical journals was significantly higher than that of the top 3 neurosurgical journals $(p<0.05)$.

their objectives, whereas $74 \%$ of pragmatic surgical trials met their objectives.

\section{Discussion}

In this study, we have conducted a high-yield search of the English literature from the year 2000 onwards to identify and assess the quality of neurosurgical RCTs. This was not a systematic review of the relevant literature, and certain topics (such as pain management) were excluded. Our analysis has shown that the prevalence of neurosurgical RCTs is low in comparison with other medical fields in general. We have demonstrated areas of deficiency with regard to both the design and the reporting of neurosurgical RCTs, with blinding, allocation concealment, and justifications for sample sizes emerging as major issues. Furthermore, discrepancies between study objectives and actual design have also been identified; many studies with explanatory/mechanistic objectives failed to meet this goal with regard to their design. This information highlights some of the shortcomings in the match made between the neurosurgical specialty and RCT trials, suggesting that perhaps alternative trial designs that are complementary to
RCTs rather than serving as substitutions (such as PROS) should be considered and implemented.

The most common RCTs were in the subspecialty fields of vascular, oncological, and functional neurosurgery. This is likely both a reflection of the higher prevalence of disorders in these fields and the overlap with other medical specialties such as neurology and medical/radiation oncology. In neurooncology, no surgical RCTs pertaining to primary low-grade lesions such as meningiomas, vestibular schwannomas, or low-grade gliomas were identified. This is perhaps a reflection of both the lower prevalence and the longer time course needed to determine outcomes, compared with metastases and high-grade gliomas. While spinal disorders are relatively common in the general population, there is little overlap between neurosurgery and other medical specialties, particularly given that we had opted to not include studies on pain management and physiotherapy. Similar to previous findings, the US, Germany, and United Kingdom continue to be the greatest contributors of RCTs; this is likely a reflection of a large population base, higher standards for conducting trials, and greater resources for conducting trials. Although the number of RCTs with relevance to neurosurgery is likely higher than that found in our study, the trend of approximately 4-5 major RCTs per year without any evidence of growth, and the steady pattern noted through a comparison with prior assessments of the neurosurgical literature, ${ }^{13,27}$ is concerning. This is in stark contrast to the overall landscape in the literature in which RCTs in general are being published at an approximate rate of 200 per week, according to an estimate in the year 2000; neurosurgery clearly lags behind. ${ }^{26}$ In terms of the quality of reporting, however, similar analyses to ours have identified significant deficiencies in other specialties such as oncology ${ }_{1,24}^{15}$ gynecology,${ }^{21}$ orthopedic surgery, ${ }^{5,23}$ and plastic surgery. ${ }^{1,30}$

Bhandari and colleagues ${ }^{5}$ assessed the quality of RCTs in orthopedics using a scale different from CONSORT and found shortcomings in the quality of reporting blinding and allocation concealment. A study by Taghinia and colleagues $^{30}$ assessed the quality of RCTs in plastic surgery, using the CONSORT statement, over a 20 -year period (1986-2006) and found that the majority of the tri-

TABLE 4. Percentage of neurosurgical RCTs meeting items in the Jadad checklist*

\begin{tabular}{lccc}
\hline \multicolumn{1}{c}{ Jadad Variable } & Surgical Studies & Drug Studies & Device Studies \\
\hline Median total score, max score 5 (IQR) & $3(2-4)$ & $3(2-3)$ & $2(1.75-3)$ \\
\hline Randomization (max score 2) & & & 40 \\
\hline Randomized \& method appropriate & 60.6 & 61.5 & 60 \\
Randomized, no elaboration of method & 36.8 & 38.5 & 0 \\
Inappropriate method of randomization & 2.6 & 0 & 0 \\
\hline Blinding (max score 2) & & 0 & 40 \\
$\quad$ Blinded \& method appropriate & 10.5 & 7.7 & 60 \\
Blinded, no elaboration of method & 31.6 & 92.3 & 40 \\
Inappropriate method of blinding & 57.9 & & 60 \\
\hline Account of all patients (max score 1) & & 84.6 & 15.4 \\
\hline All patients accounted for & 92.1 & 7.9 & \\
All patients not accounted for & & & \\
\hline
\end{tabular}

* All data given as percentages unless otherwise indicated. 

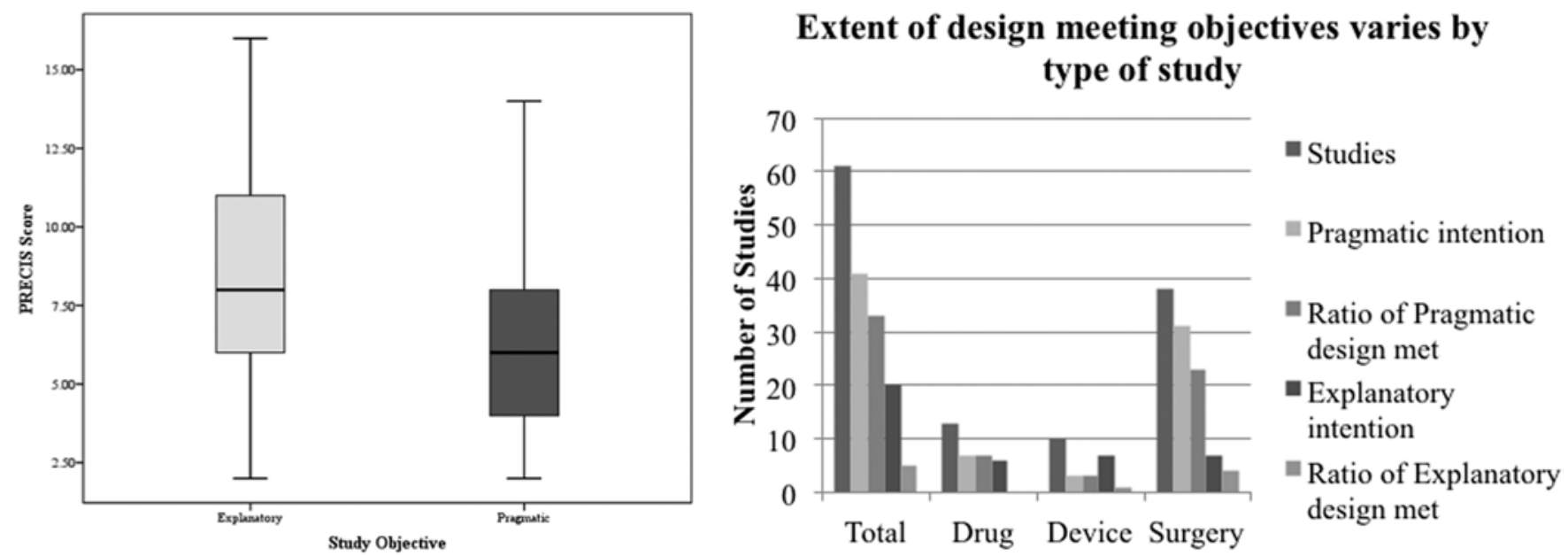

FIG. 3. Left: Graph of the median PRECIS-based score for studies stating pragmatic or explanatory objectives demonstrates substantial overlap. The horizontal line is the median. Right: The majority of studies stated pragmatic objectives, and these objectives were mainly met based on the trial design. Explanatory objectives were less successfully met.

als did not adequately report allocation concealment and sample size calculations/justifications; in fact, these fields were rated lower in this review than our assessment of neurosurgical RCTs. The quality of the methodology, assessed through the Jadad score, was low in these studies as well. Therefore, it is clear that the issue of poor quality reporting and methodology of RCTs is pervasive and applies to many medical specialties. In surgical specialties, the emerging theme appears to be the issue of sample size calculation/justification, blinding, and allocation concealment. The causes for these deficiencies are multifactorial.

Many of the concepts in surgery in general, but particularly neurosurgery, have been passed down from mentors to mentees without significant consideration of possible equipoise between alternative management strategies for an individual practitioner. Therefore, from the perspective of principle, the conduct of a trial to assess the difference between two interventions would be unethical. ${ }^{16}$ Although Freedman has argued that equipoise within the clinical community is sufficient to justify a trial,$^{10}$ the inherent bias associated with conducting a trial by individual surgeons cannot be ignored. In our assessment, two-thirds of surgical trials and more than $30 \%$ of drug/device trials did not meet any of the CONSORT criteria for reporting on blinding. While some of the trials had simply not noted their methods of blinding, a concerning majority had simply implemented an incorrect method of blinding based on the Jadad criteria. Although the quality of reporting on allocation concealment was slightly better, a minority of trials had met all of the CONSORT criteria for reporting. In a study by Kunz et al., it has been shown that observational studies and RCTs with poor allocation concealment are of equally low qualities. ${ }^{14}$ The overall CONSORT score was found to be higher in articles published in medical journals such as NEJM and JAMA. Although most neurosurgeons are aware of landmark papers in nonneurosurgical journals, the majority of our knowledge comes from the specific neurosurgical journals. The general agreement of our findings with that of Kiehna et al., ${ }^{13}$ focusing only on 2006 and 2007, not only validates the CONSORT and Jadad scales across assessors but also demonstrates that the deficiencies are not limited to a particular cohort of studies in time. The standardized number of times an RCT was cited was not correlated with its quality of reporting. While it is possible that low-quality RCTs are cited frequently for their shortcomings, it is more likely that they are being cited based on their results. This is a concerning observation, given the possibility that low-quality RCTs are resulting in an erroneously high impact on the neurosurgical literature.

It has been suggested that clinicians will tend to, consciously or subconsciously, implement their own bias toward a particular intervention. ${ }^{28}$ They may even go so far as to find it an "intellectual challenge" to identify the true allocation of a given patient. ${ }^{29}$ This pursuit, and other potential biases, can result in a breakdown of the blinding and allocation concealment of a trial that has not been vigorously designed, which ultimately can lead to either overestimating the benefit of a treatment or, on the contrary, not identifying an effect when there is one. ${ }^{14}$ Considering the fact that RCTs are known as the gold standard of evidence, incorrectly conducted studies can result in erroneous establishment of guidelines for clinical entities and management situations in a specialty that is in dire need of them.

The median sample size for all trials in our study was relatively low, with the majority of studies based on samples smaller than 100. In contrast to conditions in other medical specialties addressed through RCTs, such as infectious diseases and chronic illnesses, the neurosurgical specialty is confronted with relatively more rare conditions. Combined with the fact that clinical epidemiologists consider larger RCTs with narrower confidence intervals to be of greater importance and value, many neurosurgical questions are either not amenable to an RCT or their quality is considered too low to be of much value. ${ }^{34}$

It may be argued that neurosurgical RCTs should be based on more rigorous design, and neurosurgical jour- 
nals, the primary source of knowledge for the neurosurgical community, should enforce reporting guidelines such as CONSORT.13 In one of the most rigorously designed RCTs applicable to neurosurgery, the North American Symptomatic Carotid Endarterectomy Trial (NASCET), ${ }^{20}$ surgeons selected to participate were screened based on their perioperative morbidity and mortality rates prior to participation. While this ensures patient safety within the trial and meets the ethical objectives of research, the findings of such a design may not be generalizable to every neurosurgeon performing a carotid endarterectomy in the community. Furthermore, a well-designed RCT requires a significant investment of essential resources. The overall time lag between the inception of a trial idea and the publication of its individual or pooled results can be significant, potentially limiting the applicability of its findings at the time of publication. ${ }^{26}$

The concern regarding the time lag from inception of an idea (such as a novel device or a new surgical technique) to its establishment as the standard of care should not be interpreted as a need to forego rigorous criteria, such as those demanded by the US FDA, Health Canada, and the European Medicines Agency. These safeguards have been established to deliver high-quality care with patient safety as the highest priority. Such safety and efficacy studies are most appropriately addressed through explanatory RCTs, and other designs are likely to be a compromise in quality. An example of an explanatory RCT design that has significantly impacted the standard of care for carotid stenosis has been the NASCET trial (not in our cohort)..$^{20}$ Once a device or a surgical technique has been approved by the FDA (as an American example), however, other agencies such as the Centers for Medicare and Medicaid Services demand the evidence that highlights patient-important outcomes, cost-effectiveness, and relevance to daily practice. To provide such evidence in favor of an intervention's effectiveness, a pragmatic design objective would be more appropriate.

The majority of the studies identified in our search were judged to have a pragmatic objective. Based on a PRECIS-based scale, many of these studies were designed to meet their objectives while the cohort of studies with explanatory objectives was generally not designed for this purpose. While it is recognized that the pragmaticexplanatory concept is a continuum rather than a dichotomous variable, our observation likely reflects the general objective and reality of neurosurgical trials in that they tend to have a pragmatic objective and are better suited to meet that objective. Some of these pragmatic studies (such as STICH) have indeed affected the practice of neurosurgery pertaining to the surgical management of spontaneous intracranial hematomas. ${ }^{18}$ However, with obvious exceptions, successfully devising a protocol that appears to effectively meet the objectives does not imply that the methodology and reporting quality of these PCTs are not deficient. Considering the term "pragmatic" as a strictly methodological definition applicable to any design that is practical and reflective of the true landscape of the field, a well-designed and implemented pragmatic observational study would be a realistic adaptation to the issue relevant to RCTs in various surgical specialties, including neuro- surgery. Combined with the aforementioned shortcomings of PCTs and traditional RCTs, it may be beneficial to consider PROS as a design that is better suited to a great proportion of neurosurgical questions, and therefore to consider it as a complementary approach when RCTs are not feasible.

Through the implementation of registries, PROS can be particularly helpful in establishing the effectiveness of a given intervention or to provide surveillance of harm in the case of rare events; this would provide relevant evidence to the Centers for Medicare and Medicaid Services and equivalent agencies. The widespread acceptance of prospective observational studies has led to the establishment of guidelines for the design of prospective registries. ${ }^{11}$ Furthermore, criteria for the design and evaluation of prospective observational studies have also been established. ${ }^{4}$ Registries that are amenable to multicenter studies are particularly helpful.

Multicenter PROS enable the analysis of outcome from a greater pool of patients who are managed based on the routine practice of the selected centers. This approach overcomes some of the inherent limitations of randomizing a large sample of patients for an RCT. Furthermore, this design seeks to obtain answers that are relevant to daily practice given that the typical patient is "enrolled" rather than the ideal patient. Depending on the particular question, centers are selected based on the interventions offered: each center would have the flexibility of offering the intervention it is most equipped and/or trained for, rather than one that they are not familiar with or do not prefer. Therefore, this approach would minimize preferential patient enrollment or outcome assessment. It is evident that this strategy does not apply to questions addressing the effect of an intervention compared with placebo. Furthermore, as with other nonrandomized studies, the lack of randomization of patients and blinding in PROS can introduce bias. Furthermore, the practice at various institutions can be greatly heterogeneous. ${ }^{35}$ Therefore, strategies must be implemented to improve this methodology.

The deviation of the concept of PROS from PCTs and traditional RCTs should not be interpreted as the former not being adherent to any quality-control guidelines. First and foremost, the investigators involved in the study must establish proof that the current question cannot be reasonably addressed through a well-designed and implemented RCT; this includes the concepts of equipoise, blinding, resource availability, and other aforementioned concerns. Furthermore, other factors such as the required sample size, planned statistical analyses, and clearly defined objectives and end points should be established upfront. These and other considerations have been comprehensively outlined in the 2012 International Society for Pharmacoeconomics and Outcomes Research (ISPOR) position statement. ${ }^{4}$ The ISPOR group further suggests that a detailed and accurate protocol be established and registered upfront to increase the validity of the study and its findings. A commitment to publishing the results, regardless of whether a positive or negative outcome was attained, is also essential. ${ }^{25}$ In addition, guidelines must be implemented to track, report, and act upon all adverse events. Protocols for the protection of patient privacy are also essential. Furthermore, while 
patients enrolled at each participating center to take part in the study are not randomized, there are no restrictions on randomizing participating centers to determine the ones that are to be included in the final analysis. Under ideal circumstances, this strategy would balance some of the baseline characteristics of the centers involved. The results of this ideal scenario would reflect a realistic pool of typical patients managed for a particular condition under routine intervention settings. The success of this approach would depend on the involvement of a large number of centers, which is not always feasible. Such strategies would be particularly relevant for addressing clinical queries pertaining to pathologies for which there is a dearth of RCTs, such as meningiomas and vestibular schwannomas.

The current study was not a systematic review of all neurosurgical RCTs in the stated time interval and it is likely that some studies may not have been included. Furthermore, the CONSORT, Jadad, and PRECIS-based scales involve subjective assessments. In addition, the PRECIS-based scale was arbitrary and this approach has not been attempted to assess the design of other studies. However, the purpose of this study was to determine the general trend of neurosurgical RCTs with regard to the quality of design and reporting, and correlate these with previous assessments. Furthermore, the CONSORT scores were assessed by 3 independent reviewers with a high correlation, and the corroboration by previous studies is reassuring as well.

The current study does not imply that RCTs are not applicable to neurosurgery and should therefore be replaced by PROS. Rather, our objective has been to demonstrate that not all neurosurgical questions can be answered through the conduct of an RCT and that alternative complementary approaches must be considered. Furthermore, we have provided strategies on developing prospective observational trials that adhere to as many of the principles of sound clinical research as possible and convey high-quality clinical findings that are applicable to routine daily practice in situations where a well-designed RCT is simply not feasible. The pace and quality of neurosurgical research can be improved.

\section{References}

1. Agha RA, Camm CF, Doganay E, Edison E, Siddiqui MR, Orgill DP: Randomised controlled trials in plastic surgery: a systematic review of reporting quality. Eur J Plast Surg 37:55-62, 2014

2. Akobeng AK: Understanding randomised controlled trials. Arch Dis Child 90:840-844, 2005

3. Baigent $\mathrm{C}$ : The need for large-scale randomized evidence. $\mathbf{B r}$ J Clin Pharmacol 43:349-353, 1997

4. Berger ML, Dreyer N, Anderson F, Towse A, Sedrakyan A, Normand SL: Prospective observational studies to assess comparative effectiveness: the ISPOR good research practices task force report. Value Health 15:217-230, 2012

5. Bhandari M, Richards RR, Sprague S, Schemitsch EH: The quality of reporting of randomized trials in the Journal of Bone and Joint Surgery from 1988 through 2000. J Bone Joint Surg Am 84-A:388-396, 2002

6. Buesching DP, Luce BR, Berger ML: The role of private industry in pragmatic comparative effectiveness trials. J Comp Eff Res 1:147-156, 2012

7. Byrne BJ, Kishnani PS, Case LE, Merlini L, Müller-Felber
W, Prasad S, et al: Pompe disease: design, methodology, and early findings from the Pompe Registry. Mol Genet Metab 103:1-11, 2011

8. Dickersin K, Scherer R, Lefebvre C: Identifying relevant studies for systematic reviews. BMJ 309:1286-1291, 1994

9. Evans D: Database searches for qualitative research. J Med Libr Assoc 90:290-293, 2002

10. Freedman B: Equipoise and the ethics of clinical research. $\mathbf{N}$ Engl J Med 317:141-145, 1987

11. Gliklich R, Campion FX: Patient registries. MGMA Connex 10:15-17, 2010

12. Jadad AR, Cook DJ, Browman GP: A guide to interpreting discordant systematic reviews. CMAJ 156:1411-1416, 1997

13. Kiehna EN, Starke RM, Pouratian N, Dumont AS: Standards for reporting randomized controlled trials in neurosurgery. $\mathbf{J}$ Neurosurg 114:280-285, 2011

14. Kunz R, Lelgemann M, Schneider M: [Evidence-based guidelines-what is the present situation?] $\mathbf{Z}$ Rheumatol 61 (Suppl 2):II/10-II/13, 2002 (Ger)

15. Liberati A, Himel HN, Chalmers TC: A quality assessment of randomized control trials of primary treatment of breast cancer. J Clin Oncol 4:942-951, 1986

16. Lilford RJ, Jackson J: Equipoise and the ethics of randomization. J R Soc Med 88:552-559, 1995

17. McCulloch P, Taylor I, Sasako M, Lovett B, Griffin D: Randomised trials in surgery: problems and possible solutions. BMJ 324:1448-1451, 2002

18. Mendelow AD, Gregson BA, Fernandes HM, Murray GD, Teasdale GM, Hope DT, et al: Early surgery versus initial conservative treatment in patients with spontaneous supratentorial intracerebral haematomas in the International Surgical Trial in Intracerebral Haemorrhage (STICH): a randomised trial. Lancet 365:387-397, 2005

19. Moher D, Schulz KF, Altman DG: The CONSORT statement: revised recommendations for improving the quality of reports of parallel-group randomized trials. J Am Podiatr Med Assoc 91:437-442, 2001

20. North American Symptomatic Carotid Endarterectomy Trial Collaborators: Beneficial effect of carotid endarterectomy in symptomatic patients with high-grade carotid stenosis. $\mathbf{N}$ Engl J Med 325:445-453, 1991

21. Partsinevelou A, Zintzaras E: Quality of reporting of randomized controlled trials in polycystic ovary syndrome. Trials 10:106, 2009

22. Pastorino U: Lung cancer screening. Br J Cancer 102:16811686, 2010

23. Poolman RW, Struijs PA, Krips R, Sierevelt IN, Lutz KH, Bhandari M: Does a "Level I Evidence" rating imply high quality of reporting in orthopaedic randomised controlled trials? BMC Med Res Methodol 6:44, 2006

24. Rada G, Schünemann HJ, Labedi N, El-Hachem P, Kairouz VF, Akl EA: Systematic evaluation of the methodology of randomized controlled trials of anticoagulation in patients with cancer. BMC Cancer 13:76, 2013

25. Roche N, Reddel H, Martin R, Brusselle G, Papi A, Thomas $\mathrm{M}$, et al: Quality standards for real-world research. Focus on observational database studies of comparative effectiveness. Ann Am Thorac Soc 11 (Suppl 2):S99-S104, 2014

26. Sackett DL, Hoey J: Why randomized controlled trials fail but needn't: a new series is launched. CMAJ 162:1301-1302, 2000

27. Schöller K, Licht S, Tonn JC, Uhl E: Randomized controlled trials in neurosurgery-how good are we? Acta Neurochir (Wien) 151:519-527, 2009

28. Schulz KF, Grimes DA: Allocation concealment in randomised trials: defending against deciphering. Lancet 359:614-618, 2002

29. Schulz KF, Grimes DA: Blinding in randomised trials: hiding who got what. Lancet 359:696-700, 2002 
30. Taghinia AH, Liao EC, May JW Jr: Randomized controlled trials in plastic surgery: a 20 -year review of reporting standards, methodologic quality, and impact. Plast Reconstr Surg 122:1253-1263, 2008

31. Thorpe KE, Zwarenstein M, Oxman AD, Treweek S, Furberg $\mathrm{CD}$, Altman DG, et al: A pragmatic-explanatory continuum indicator summary (PRECIS): a tool to help trial designers. CMAJ 180:E47-E57, 2009

32. Urschel JD, Urschel DM, Miller JD, Bennett WF, Young JE: A meta-analysis of randomized controlled trials of route of reconstruction after esophagectomy for cancer. Am J Surg 182:470-475, 2001

33. Vranos G, Tatsioni A, Polyzoidis K, Ioannidis JP: Randomized trials of neurosurgical interventions: a systematic appraisal. Neurosurgery 55:18-26, 2004

34. Yusuf S, Collins R, Peto R: Why do we need some large, simple randomized trials? Stat Med 3:409-422, 1984

35. Yusuf S, Garg R, Zucker D: Analyses by the intention-totreat principle in randomized trials and databases. Pacing Clin Electrophysiol 14:2078-2082, 1991

36. Zwarenstein M, Treweek S, Gagnier JJ, Altman DG, Tunis S, Haynes B, et al: Improving the reporting of pragmatic trials: an extension of the CONSORT statement. BMJ 337:a2390, 2008

\section{Disclosure}

The authors report no conflict of interest concerning the materials or methods used in this study or the findings specified in this paper.

\section{Author Contributions}

Conception and design: Kondziolka, Mansouri. Acquisition of data: Mansouri, Cooper, Shin. Analysis and interpretation of data: Mansouri, Cooper, Shin. Drafting the article: Kondziolka, Mansouri. Critically revising the article: Kondziolka, Mansouri. Reviewed submitted version of manuscript: Kondziolka, Mansouri. Statistical analysis: Mansouri. Study supervision: Kondziolka.

\section{Correspondence}

Douglas Kondziolka, Departments of Neurosurgery and Radiation Oncology, Suite 8R, NYU Langone Medical Center, New York, NY 10016. email: douglas.kondziolka@nyumc.org. 